\title{
Propagación de arboles de teca Tectona Grandis L. f. por miniestacas
}

\author{
Propagation of Tectona Grandis L. f. trees by minicutting
}

\author{
Andrés R. Meza ${ }^{1 *}$, Javier Rodriguez² ${ }^{2}$ Kellen C. Gatti ${ }^{3}$, Erika E. Espinoza ${ }^{4}$. \\ Recibido para publicación: Mayo 25 de 2015 - Aceptado para publicación: Noviembre 27 de 2015
}

\begin{abstract}
RESUMEN
La propagación vegetativa en forestales permite producir plantaciones homogéneas con características fenotípicas deseables en corto tiempo. Este trabajo tuvo como objetivo evaluar la propagación de árboles de teca, usando la técnica de miniestacas. La investigación se realizó en el municipio de Canalete, Córdoba, Colombia, en rodales comerciales de teca de 13 a 17 años de edad, en los cuales se tomaron estacas del tercio inferior de la copa, de $30 \mathrm{~cm}$ de longitud con dos a cuatro brotes, las cuales se plantaron en un sustrato de arena y aluvión. Cuando los brotes o miniestacas alcanzaron una longitud de dos a cinco $\mathrm{cm}$, se cortaron y se les aplicó tratamientos de enraizamiento, con ácido indolbutirico AIB y polivinilpirrolidona PVP en las siguientes dosis: Testigo(00:00), T1(2000:4000), T2(2000:8000), T3(2000:16000), T4(3000:4000), T5(3000:8000), T6(3000:16000), T7(5000:4000) y T8(5000:16000) mg $\mathrm{L}^{1}$; posteriormente se plantaron en turba, en un diseño completamente al azar con cuatro repeticiones. Se evaluaron los porcentajes de supervivencia en cámara húmeda, enraizamiento en casa sombra y número de raíces. Los resultados mostraron diferencias altamente significativas entre los tratamientos para porcentajes de supervivencia en cámara húmeda y enraizamiento en casa sombra con respecto al testigo, siendo los tratamientos T4(3000:4000) y T8(5000:16000) más eficientes, con valores superiores al 55 y $40 \%$ respectivamente. El mayor número de raíces se obtuvo con el tratamiento T7(5000:4000), con 2,5 raíces por brote. Se concluye que la técnica de miniestaca es una metodología potencial en la propagación de árboles de teca de alto valor comercial.
\end{abstract}

Palabras claves: Clonación, miniestacas, propagación, Teca.

\begin{abstract}
Vegetative propagation in forests allows for producing homogeneous plantations with desirable phenotypic characteristics over a short period of time. The purpose of this study was to assess the propagation of teak trees by using minicutting. The study took place at Canalate Córdoba, Colombia, in 13 to 17 year old commercial teak stands, where $30 \mathrm{~cm}$ cuttings from the lower third of the treetop with two to four sprouts were taken and subsequently planted in a sand and alluvium substrate. Once these cuttings reached a 2-to-5-cm length, they were cut and later were applied rooting treatments mixed with IBA (indole butyric acid) and PVP (polivinilpirrolidona) in the following doses: Control(00:00), C1(2000:4000), C2(2000:8000), C3(2000:16000), C4(3000:4000), C5(3000:8000), C6(3000:16000), C7(5000:4000) y C8(5000:16000) $\mathrm{mg} \mathrm{L}^{\cdot 1}$. They were later planted in a peat, in a random design with four replications. Percentages of survival in a moist chamber $(\% \mathrm{SCH})$, rooting shade house (\% ECS), and the number of roots were evaluated. Results showed highly significant differences between treatments for rates of survival in humid chamber, and rooting in shade house with regard to the control, being the treatments C4(3000:4000) and C8(5000:16000) more efficient with values over 55 and $40 \%$ respectively. Regarding the number of roots, the highest number was obtained in the C7(5000:4000) treatment with 2,5 roots per sprout. It can be concluded that minicutting is a potential technique for the propagation of Tectonagrandis Linn F trees with high commercial value.
\end{abstract}

Key words: Cloning, Rooting minicuttings, clonal propagation, Tectona grandis, hormones and antioxidant

\footnotetext{
1*Ingeniero Agrónomo, investigador, CONIF. Calle 72, Numero 12 - 67 Bogotá; teléfono: (1) 2118020; correo electrónico: andresrafael2@ hotmail.com

${ }^{2}$ Ingeniero Forestal, director de proyectos, CONIF.

${ }^{3}$ Ingeniera Forestal, asesora de proyectos, Universidad de Córdoba.

${ }^{4}$ Ingeniera Agrónoma, investigador, CONIF.
} 


\section{INTRODUCCIÓN}

El objetivo de un programa de mejoramiento genético, es obtener las principales características cuantitativas y cualitativas a través de la selección progresiva de genes deseables y su perpetuación mediante la utilización de semillas o de clones mejorados. La clonación es entonces una herramienta o el primer paso en un programa de mejoramiento, mediante el cual se captura de forma rápida la mayor proporción de la variación genética y como consecuencia, se maximizan los progresos provenientes de la selección en cada ciclo de mejoramiento (Carpineti 2005).

En programas de silvicultura clonal, la selección del árbol superior es generalmente realizada en la fase adulta; luego se multiplica asexualmente, mediante diferentes procesos de brotación y enraizamiento (Alfenas et al. 2004). La propagación vegetativa de árboles adultos requiere de material vegetal fisiológicamente joven, ya sea por la utilización de partes jóvenes de la planta (yemas epicórmicas basales) o por la formación de estacas jóvenes en partes de la planta adulta, restaurando la competencia o enraizamiento. La importancia de usar material joven en la propagación es estimular el efecto ortotrópico presentado por estos; ya que los materiales adultos son más propensos al efecto plagiotrópico, que no es deseable en el proceso de clonación (Gatti et al. 2011).

La forma más común de rescate o revigorización de los arboles adultos es la tala, para así inducir la emisión de brotes basales. Existen otras formas de revigorizar el árbol como son el anillamiento del tallo, la inducción de brotes en ramas podadas, la aplicación de fuego, el rescate por injerto o micropropagación; sin embargo, debe tenerse en cuenta que las especies responden de manera diferente a las técnicas de rescate (Alfenas et al. 2004).
La propagación por miniestaca surgió a partir de las limitaciones de la microestaca (propagación in vitro) con relación al aspecto operacional, técnico y económico, y consiste en la utilización de brotes de plantas propagadas por el método de la estaca convencional, así como de plántulas producidas por la propia miniestaca o de semillas, como fuente de propágulos vegetativos para propagación clonal masal (Alfenas et al. 2004; Assis y Mafia 2007; Xavier 2009). Otros autores han utilizado con éxito la miniestaca como técnica para enraizamiento de árboles adultos (Gatti 2002; Monteuuis y Maître 2007; Murillo y Badilla 2009; Leiva 2010; Gatti et al. 2011; Husen 2013).

Goulart et al. (2010), indican que la aplicación de hormonas y antioxidantes, en la propagación de miniestacas permite aumentar los porcentajes de supervivencia en los procesos de propagación. Soto (2004), menciona que el ácido indolbutirico AIB es la mejor hormona para el uso masivo en enraizamiento, ya que en una gama amplia de concentraciones no genera fitotoxicidad y es efectivo para un gran número de especies. Para el caso del uso del polivinilpirrolidona PVP Gatti et al. (2011), menciona que produce un efecto antioxidante el cual consiste en la inactivación de los radicales libres, en el complejo de iones metabólicos en una reducción de los peróxidos, disminuyendo la formación de estos radicales con potencial de oxidación.

Según Espitia et al. (2011) en el departamento de Córdoba, Colombia, se proyecta plantar 200.000 ha forestales para el 2025, siendo la teca una de las especies preferidas en los planes de reforestación para el departamento, donde se encuentran árboles de más de 20 años. La madera de teca es de alta calidad con un alto valor en los mercados internacionales ha motivado su siembra en diferentes regiones tropicales de América (Trujillo 2009; Ladrach 2010). Entre los limitantes de la reforestación en Córdoba, está la poca disponibilidad de 
semilla forestal certificada, planteándose como objetivo de este trabajo evaluar la propagación de árboles seleccionados de teca, por la técnica de miniestacas, como una opción de fomento en la reforestación clonal.

\section{MATERIALES Y MÉTODOS}

Este experimento fue realizado en el vivero de producción de plántulas de Tectona grandis, en el corregimiento del Limón, Municipio de Canalete, Córdoba, localizado a $8^{\circ} 37^{\prime} 46,3^{\prime \prime}$ $\mathrm{N}$ y $76^{\circ} 15^{\prime} 04,3^{\prime \prime}$ W. La región se caracteriza por poseer un clima seco tropical, con una precipitación anual de $1552 \mathrm{~mm}$, temperatura media de $24,3^{\circ} \mathrm{C}$, (Palencia et al. 2006).

\section{Inducción de miniestacas}

Se seleccionaron arboles de Tectona grandis entre los 13 y 17 años, de los cuales se colectaron ramas provenientes del tercio inferior de la copa; cortando estacas de $30 \mathrm{~cm}$ de largo. Con el fin de evitar la deshidratación de las estacas, estas se almacenaron envueltas en papel servilleta y se trasportaron en una cava con hielo, teniendo la precaución de separar las estacas del hielo con una cobertura de cascarilla de arroz previamente desinfectada con fungicida; posteriormente se trasladaron a las instalaciones del invernadero donde se sembraron en un sustrato compuesto por arena y aluvión en una proporción 1:1. Se aplicaron riegos manuales tres veces al día, 7 am, 2 y 5 pm; adicionalmente, con el fin de inducir la aparición de brotes epicórmicos (miniestacas), cada ocho días se realizaron aplicaciones de PROGIB $^{\circledR}, 4 \mathrm{mg} \mathrm{L}^{-1}$, en el riego de 5 de la tarde.

\section{Supervivencia y enraizamiento de las miniestacas \\ Después de 45 días de plantadas las estacas en el sustrato, se colectaron los brotes epicórmicos o miniestacas de 2 a $5 \mathrm{~cm}$ de largo y 0,5-1 cm de diámetro, con dos entrenudos y máximo dos hojas, de las cuales se les retiró el 70\% de la lámina foliar. Estas miniestacas fueron sumergidas su base}

$(0,5 \mathrm{~cm})$ durante 10 segundos aproximadamente en tratamientos de enraizamiento con AIB (ácido indolbutirico) y PVP (polivinilpirrolidona) en las siguientes combinaciones respectivamente en mg L ${ }^{1}$ : Testigo (00:00), T1(2000:4000), T2(2000:8000), T3(2000:16000), T4(3000:4000), T5(3000:8000), T6(3000:16000), T7(5000:4000) у T8(5000:16000).

Una vez tratadas las miniestacas se plantaron en sustrato, (turba en forma de pellets y posteriormente hidratada) y se llevaron a cámara húmeda por 30 días, y posteriormente 10 días en patio de enraizamiento o casa sombra; después se trasladaron al patio de rustificación a pleno sol. Las prácticas culturales como riego, nutrición y control fitosanitario fueron las descritas por Gatti et al. (2011).

Los experimentos se realizaron bajo un diseño completo al azar, con nueve tratamientos y cuatro repeticiones; donde cada unidad experimental estaba conformada por cuatro miniestacas. Se evaluó el porcentaje de supervivencia en cámara húmeda $\mathrm{SCH}$, el porcentaje de enraizamiento en casa sombra ECS y el número de raíces NR a los 40 días de edad.

Para el caso del NR, se contaron las raíces adventicias que se produjeron en la base de las diferentes miniestacas. Los datos recolectados para cada variable fueron tabulados con el programa Excel y posteriormente se realizó un análisis de varianza y una prueba de comparación de medias (Tukey) con el programa S.A.S. Versión 9.1.

\section{RESULTADOS Y DISCUSIÓN}

\section{Inducción de miniestacas}

Las estacas iniciaron la producción de brotes a los 15 días después de plantadas en el sustrato, coincidiendo con los resultados de Gatti et al. (2011) En otras especies como el caso de la guadua (Guadua angustifolia), la inducción de 
brotes (miniestacas) inició a partir del quinto día (Gallardo et al. 2008), corroborando que los brotes producidos se encuentran en función de la especie, el genotipo, grado de desarrollo de la planta donadora, y los factores ambientales como la luz, temperatura y humedad, como también el manejo al material después de ser cosecha y establecido en el sustrato (Gatti et al. 2011).

\section{Supervivencia y enraizamiento de las miniestacas}

Los porcentajes de supervivencia de las miniestacas en cámara húmeda, estuvieron entre 87,5 y $32,34 \%$; mientras que, en la casa sombra estuvieron entre 69,39 y 29,34\%. Tanto en cámara húmeda como en casa de sombra el porcentaje de mortalidad mostró diferencias altamente significativas entre los tratamientos, obteniéndose los mayores porcentajes de sobrevivencia en los tratamientos T4(3000:4000), T6(3000:16000), T7(5000:4000) y T8(5000:16000) (Tabla 1).

Los resultados obtenidos en esta investigación, son considerados pioneros en la región, ya que no se encontraron reportes de propagación de teca por la técnica de miniestacas, como tampoco el uso de la mezcla de AIB y PVP para enraizamiento. La literatura consultada reporta resultados del uso individual de AIB para enraizamiento en diferentes especies forestales, tales como Eucalyptus grandis x E. urophylla (Goulart et al. 2010); paricá (Schizolobium amazonicum (Rosa y Pinheiro 2001) y AcaciaNegra Acacia mearnsii Willd ( Borges y Martins 2002), los cuales afirman que el incremento en la concentración de la hormona, ocasiona un incremento en el porcentaje de enraizamiento.

\section{Producción de raíces}

El análisis de varianza mostró diferencias significativas para la variable número de raíces NR, la prueba de Tukey indicó que el tratamiento T7(5000:4000) produjo un mayor número de raíces, (Tabla 1). Santos et al. (2006), probando diferentes dosis de AIB, acompañada de una dosis constante de PVP, encontraron diferencias en la producción del número de raíces en estaquillas de Caryocar brasiliense Camb.

Tabla 1. Efecto de la combinación de ácido indolbutirico AIB y polivinilpirrolidona PVP, en el número de raíces NRA, porcentaje de supervivencia en cámara húmeda $\mathrm{SCH}$ y porcentaje de enraizamiento en casa sombra ECS, de árboles seleccionados de Tectona grandis.

\begin{tabular}{lccc}
\hline $\begin{array}{c}\text { TRATAMIENTOS } \\
\text { (AIB:PVP) }\end{array}$ & NRA & \multicolumn{1}{c}{ SCH(\%) } & \multicolumn{1}{c}{ ECS(\%) } \\
\hline Testigo(00:00) & $1,00 \pm 1,01 \mathrm{ab} *$ & $32,34 \pm 4,01 \mathrm{~d}$ & $29,34 \pm 10,60 \mathrm{~b}$ \\
T1(2000: 4000) & $1,35 \pm 0,34 \mathrm{ab}$ & $55,02 \pm 4,10 \mathrm{bcd}$ & $46,27 \pm 10,34 \mathrm{ab}$ \\
T2(2000: 8000) & $1,70 \pm 0,38 \mathrm{ab}$ & $34,58 \pm 12,75 \mathrm{~d}$ & $35,16 \pm 9,90 \mathrm{~b}$ \\
T3(2000: 16000) & $0,60 \pm 0,71 \mathrm{~b}$ & $49,26 \pm 6,96 \mathrm{~cd}$ & $44,24 \pm 9,86 \mathrm{ab}$ \\
T4(3000: 4000) & $1,12 \pm 1,03 \mathrm{ab}$ & $87,50 \pm 8,77 \mathrm{a}$ & $63,33 \pm 12,84 \mathrm{a}$ \\
T5(3000: 8000) & $1,35 \pm 0,94 \mathrm{ab}$ & $35,26 \pm 10,67 \mathrm{~d}$ & $37,12 \pm 5,50 \mathrm{~b}$ \\
T6(3000:16000) & $0,97 \pm 0,89 \mathrm{~b}$ & $59,92 \pm 10,56 \mathrm{bc}$ & $54,56 \pm 9,60 \mathrm{ab}$ \\
T7(5000: 4000) & $2,55 \pm 0,10 \mathrm{a}$ & $71,87 \pm 11,40 \mathrm{ab}$ & $51,09 \pm 6,99 \mathrm{ab}$ \\
T8(5000:16000) & $1,10 \pm 0,11 \mathrm{ab}$ & $82,10 \pm 6,33 \mathrm{a}$ & $69,39 \pm 17,80 \mathrm{a}$ \\
\hline
\end{tabular}

*Letras minúsculas distintas en sentido vertical indican diferencias significativas según la prueba de Tukey. 
Barcelo et al. (2001) argumentaron que al aumentar la concentración de auxinas también se aumentó el número de raíces, hasta llegar a un punto máximo donde el aumento de la concentración ocasiona un decremento en la producción de raíces, autores como el caso de Murillo y Badilla (2012) expresan que las concentraciones de auxinas exógenas aplicadas para procesos de enraizamiento no deben ser superiores a $10000 \mathrm{mg} \mathrm{L}^{-1}$; igualmente señalan que en especies forestales como Gmelina arborea, Eucalyptus Spp, Cupressus lusitanica y Alnus acuminata entre otras requieren una dosis aproximada de $2000 \mathrm{mg} \mathrm{L}^{-1}$.

Ya finalizado el proceso de evaluación, las plántulas obtenidas por miniestacas se trasportaron al patio de rustificacion, donde se plantaron a pleno sol y siendo empleadas actualmente como plantas madres.

\section{CONCLUSIONES}

Los resultados evidencian que la regeneración de árboles seleccionados de Tectona grandis por la técnica de miniestacas, tiene un alto potencial para la clonación de la especie. Adicional a esto se pudo determinar que el uso de hormonas y antioxidantes como el AIB y el PVP, incrementaron en relación al testigo la supervivencia en cámara húmeda hasta 55\% y el enraizamiento encasa de sombra hasta $40 \%$.

\section{AGRADECIMIENTOS}

A la empresa Ganados y Maderas, por la disponibilidad del material experimental, físico y de personal de apoyo; al Departamento Administrativo de Ciencia, Tecnología e Innovación (COLCIENCIAS) y a la Corporación nacional de investigación y fomento forestal (CONIF) por suporte técnico y financiero.

\section{REFERENCIAS}

Alfenas, A., Zauza, E., Mafia, R. y Assis, T. 2004. Clonagem e doenças do eucalipto, Universidade Federal de Viçosa. Viçosa. $442 \mathrm{p}$

Assis, T. y Mafia, R. 2007. Hibridação e clonagem, In: A. BORÉM (ed). Biotecnologia Florestal. Viçosa: [s.n.]: 93-121.

Barceló, J., Nicolás, G., Sabater, B. y Sanchéz, R. 2001. Fisiología vegetal. Ediciones Pirámide, Madrid, España: p566.

Borges, N. y Martins, M. 2002. Efeito do ácido indolbutírico no enraizamiento de estacas de acacia negra (Acacia mearnsii De Wild.). Revista Árvore, 26(2): 223227.

Goulart, P., Xavier, A. y Moreira, J. 2010. Efeito de antioxidantes no enraizamiento de miniestacas de clones de Ecualyptus grandis $\times$ E. urophylla. Árvore, 34(6): 961-972

Carpineti, L. 2005. Importancia de la silvicultura clonal. Revista IDIA XXI. 8(1): 147-150.

Espitia, M. Murillo O. Castillo C. 2011. Ganancia genética esperada en teca (Tectona grandis I.f.) en córdoba (Colombia). Colombia forestal. 4(1): 8193.

Gallardo, J., Freire, M., León, J., García, Y. Pérez, S. y Gonzáles M. 2008. Comportamiento en la brotación de las yemas de estacas de Guadua angustifolia Kunt. Empleadas en la propagación. Cultivos tropicales, 29(1): 17-22

Gatti, K. 2002. Propagação vegetativa de pau mulato (Calycophyllum spruceanum (Benth) K. Schum.), jequitibá (Cariniana estrellensis (Raddi) Kuntze) e teca (Tectona grandis Linn. f.) por miniestaquia. Tesis (maestria em Ciencias Florestais). 
Departamento de Engenharia Florestal. Universidade Federal de Viçosa, Viçosa, MG 72p.

Gatti, K., Suarez, I., Espitia, M. y Tobar, D. 2011. Producción De Plántulas Por Miniestacas de Tectona grandis Linn F., Acacia mangium Wild y Gmelina arbórea Roxb. Universidad de Córdoba, Montería - Colombia, 75p.

Hartmann, H., Kester, D., Davies, F. y Geneve, R. 2002. Plant propagation: Principles and Practice. 7th ed. Prentice Hall Inc. New Jersey, USA. 880 p.

Husen, A. Clonal Multiplication of Teak (Tectona grandis) by Using Moderately Hard Stem Cuttings: Effect of Genotypes (FG1 and FG11 Clones) and IBA Treatment. Advances in Forestry Letters (AFL) Volume, 2.

Ladrach, W. 2010. Expansion of pulp production in the third world. Versión en línea: http:// www.alleghenysaf.org/winter_2010. [ 26 de febrero del 2010]

Latsague, M., Sáes, P. y Hauenstein E. 2008. Inducción de enraizamiento en estacas de Berberidopsis corallina con ácido indolbutírico, BOSQUE, 29(3): 227-230.

Monteuuis, O. y Maître, H. 2007. Adelantos en la clonación de teca: Los ultimos avances realizados en la clonacion de teca permiten mejorar la calidad del material de plantación. OIMT Actualidad forestal tropical, 17 (3) : 13-15.

Murillo, O. y Badilla, Y. 2009. Reproducción clonal de árboles. Taller de Publicaciones. Instituto Tecnológico de Costa Rica, ITCR. Cartago. 45 p.

Palencia, G., Mercado, T. y Combatt E. 2006. Estudio Agroclimático del Departamento de Córdoba. Universidad de Córdoba. Montería, Colombia: 126p.
Santos, B., Paiva, R., Nogueira, R, Oliveira, L., Silva, D., Martinotto, C. y Paiva, P. 2006. Micropropagation of" pequizeiro" (Caryocar brasiliense Camb.). Revista Brasileira de Fruticultura, 28(2), 293-296.

Rosa, L., Pinheiro, K. 2001, Propagação vegetativa de estacas de paricá (Schizolobium amazonicum Huber ex Ducke) obtidas de material juvenil e imersasem ácido indol-3-butírico. Revista de Ciências Agrárias, 35(1), p.79-88.

Soto P. 2004. Reproducción vegetativa por estacas en Amomyrtus luma (luma), Amomyrtus meli (meli) y Luma apiculata (arrayán)mediante el uso de plantas madres jóvenes y adultas, Trabajo de Titulación presentado como parte de los requisitos para optaral Título de Ingeniero Forestal, Universidad Austral de Chile.

Leiva, S. 2010. Efecto de sustratos, reducción de lamina foliar y concentración de ácido naftalenacetico enraizamiento de miniestacas de teca/. Ciencia y tecnología para la competitividad del sector agropecuario 2002-2010: Resultado de algunos proyectos cofinanciados por el Ministerio de Agricultura y Desarrollo Rural, 78-79.

Trujillo, E. 2009. Guía de reforestación. Bogotá, Colombia: El Semillero. S.A. p. 61

Titon, M. 2001. Propagação clonal de Eucalyptus grandis por miniestaquia e microestaquia. Dissertação, tesis de maestria e ciências forestales-departamento de engenheira florestal, Universidade federal de Viçosa $65 p$.

Xavier, A. 2009. Silvicultura clonal: princípios e técnicas. Viçosa, MG: ed. UFV. 272 p. 